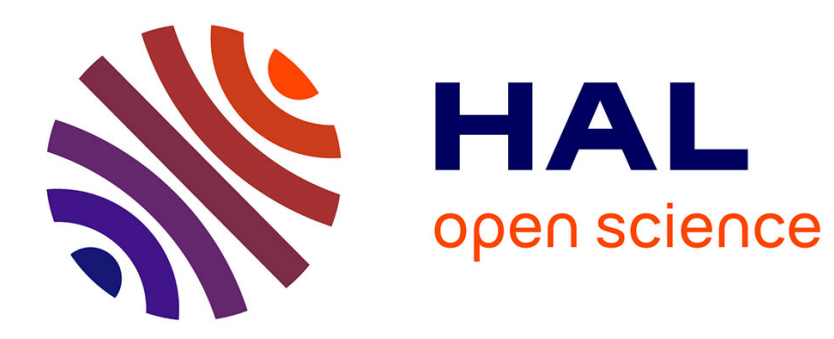

\title{
Uniformities on free semigroups
}

Jean-Eric Pin, Pascal Weil

\section{To cite this version:}

Jean-Eric Pin, Pascal Weil. Uniformities on free semigroups. International Journal of Algebra and

Computation, 1999, 9, pp.431-453. hal-00113716

\section{HAL Id: hal-00113716 \\ https://hal.science/hal-00113716}

Submitted on 14 Nov 2006

HAL is a multi-disciplinary open access archive for the deposit and dissemination of scientific research documents, whether they are published or not. The documents may come from teaching and research institutions in France or abroad, or from public or private research centers.
L'archive ouverte pluridisciplinaire HAL, est destinée au dépôt et à la diffusion de documents scientifiques de niveau recherche, publiés ou non, émanant des établissements d'enseignement et de recherche français ou étrangers, des laboratoires publics ou privés. 


\title{
Uniformities on free semigroups
}

\author{
J.-E. Pin and P. Weil \\ LIAFA \\ Université Paris- 7 and CNRS \\ 2 place Jussieu \\ 75251 Paris Cedex 05 - France. \\ \{Jean-Eric.Pin, Pascal. Weil\}@liafa.jussieu.fr
}

\begin{abstract}
Résumé. On sait que les complétions profinies d'un semigroupe libre qui sont associées à une pseudovariété de semigroupes ou de semigroupes ordonnés peuvent être définies par un écart ou un quasi-écart. Nous caractérisons les quasi-écarts et les quasi-uniformités qui peuvent être obtenues de cette façon. Nous démontrons également l'existence d'une correspondance injective "à la Eilenberg", entre pseudovariétés de semigroupes ordonnés et ce que nous appelons des variétés de quasi-uniformités.
\end{abstract}

\begin{abstract}
It is known that the profinite completions of a free semigroup which are associated with a pseudovariety of semigroups or of ordered semigroups, can be defined by an écart or a quasi-écart. We characterize those quasi-écarts and those quasi-uniformities which arise in this fashion. We also prove an Eilenberg-like one-to-one correspondence between pseudovarieties of ordered semigroups and so-called varieties of quasi-uniformities.
\end{abstract}

\section{Introduction}

Let $A^{+}$be a free semigroup. If $\mathbf{V}$ is a pseudovariety of finite semigroups, the profinite topology on $A^{+}$associated with $\mathbf{V}$ (see $[1,2]$ ) is defined by a metric $d \mathbf{V}$, as soon as it is Hausdorff. Now the question arises to know which metrics are obtained in this way.

We show that a metric on $A^{+}$is uniformly equivalent with a metric of the form $d_{\mathbf{V}}$ if and only if it satisfies the following properties (Theorem 5.10):

(1) it is ultrametric,

(2) $A^{+}$is precompact,

(3) concatenation is uniformly continuous,

(4) morphisms from $A^{+}$into $A^{+}$are uniformly continuous.

We also obtain a one-to-one correspondence, in the spirit of Eilenberg's variety theorem [8], between pseudovarieties of semigroups and metrics associated with pseudovarieties (Theorem 5.7).

Actually, slightly more general results are reached. First, there is no real mathematical reasons to limit ourselves to pseudovarieties leading to a Hausdorff profinite topology. Indeed, pseudovarieties as important as the class of finite commutative semigroups would be left out of the game. Second, recent developments in the theory of languages led to the consideration of pseudovarieties of finite ordered semigroups on the same footing as pseudovarieties of finite semigroups. For instance, it has been shown that Eilenberg's variety theorem can be extended to that case [13].

Now metrics do not suffice to fit in this larger scope. Removing the Hausdorff property amounts to eliminating the separation axiom $(d(x, y)=0$ implies $x=y)$ from the definition 
of a metric, that is, to considering écarts instead of metrics. Extending the results to ordered semigroups amounts to eliminating the symmetry axiom $(d(x, y)=d(y, x))$, that is, to considering quasi-écarts. Although it may come as a surprise to the reader that anything substantial is left after eliminating two of the three axioms of a metric, it is indeed the case.

It turns out that the proper setting to unify all these results are the so-called quasiuniform spaces. It has long been known that uniform spaces are a good abstraction of metric spaces [4]. The notion of quasi-uniform spaces has also been known for some time $[6,7,9,11]$, and it has gained a renewed interest for its recent applications in semantics $[3,16,17,19,20,21]$. So the one-to-one correspondence we establish is really between pseudovarieties of ordered semigroups and certain collections of quasi-uniformities, defined on all finitely generated free semigroups.

Let us now describe our results in a more precise way. Basic definitions are given below, in Section 2.

Every order on a semigroup defines a quasi-uniformity on that semigroup in a natural way, namely the quasi-uniformity of which the order relation is a basis. Let $\mathbf{V}$ be a pseudovariety of finite ordered semigroups and let $A$ be an alphabet. The quasi-uniformity on $A^{+}$associated with $\mathbf{V}$ is the least quasi-uniformity such that each semigroup morphism into an ordered semigroup in $\mathbf{V}$ is uniformly continuous.

It turns out that such a quasi-uniformity $\mathcal{U}$ can always be described by a quasi-écart (a precise formula is given in Section 5.3). Quasi-écarts arising in this way are characterized by conditions (1)-(4) given at the beginning of this paper. In order to characterize those quasi-uniformities without reference to quasi-écarts, it suffices to replace conditions (1) and (2) by

$\left(1^{\prime}\right) \mathcal{U}$ has a basis of transitive entourages;

$\left(2^{\prime}\right) \mathcal{U}$ is totally bounded.

(See Section 3 for the definitions).

We also characterize globally the collections of quasi-uniformities $\left(\mathcal{U}_{A}\right)_{A}$, indexed by all finite alphabets, which can be associated with a fixed pseudovariety: in addition to the conditions already mentioned, which must be imposed on each individual quasi-uniformity, one requires the additional condition below.

(5) Every morphism between free semigroups is uniformly continuous.

Such collections of quasi-uniformities (satisfying conditions $\left(1^{\prime}\right),\left(2^{\prime}\right),(3)$ and $\left.(5)\right)$ are called varieties of quasi-uniformities. The converse direction of our correspondence is as follows. Let $\left(\mathcal{U}_{A}\right)_{A}$ be a variety of quasi-uniformities. We show that the entourages of the $\mathcal{U}_{A}$ can be described in terms of rational languages of $A^{+}$, called the $\mathcal{U}_{A}$-blocks, and the associated pseudovariety is generated by the syntactic quasi-ordered semigroups of the $\mathcal{U}_{A}$-blocks.

The paper is organized as follows. Quasi-uniform spaces are defined in Section 2. Totally bounded and transitive quasi-uniformities are studied in Section 3. Semigroups (and in particular quasi-uniform semigroups) form the core of Section 4. Our main results are proved in Section 5, and several important examples are given.

\section{Quasi-uniform spaces}

This section surveys the basic definitions and results on uniform and quasi-uniform spaces which will be needed in the sequel. For more details, the reader is referred to $[4,9]$. 
Let $X$ be a set. The subsets of $X \times X$ can be viewed as relations on $X$. In particular, if $U$ and $V$ are subsets of $X \times X$, we use the notation $U V$ to denote the composition of the two relations, that is, the set

$$
U V=\{(x, y) \in X \times X \mid \exists z \in X,(x, z) \in U \text { and }(z, y) \in V\} .
$$

Given a relation $U$, the transposed relation of $U$ is the relation

$$
{ }^{t} U=\{(x, y) \in X \times X \mid(y, x) \in U\}
$$

and the symmetrized relation of $U$ is the relation

$$
U^{*}=U \cap{ }^{t} U
$$

A relation $U$ is symmetrical if ${ }^{t} U=U$. By construction, $U^{*}$ is a symmetrical relation. Finally, if $x \in X$ and $U \subseteq X \times X$, we write $U(x)$ for the set $\{y \in X \mid(x, y) \in U\}$.

\subsection{Quasi-uniformity}

A quasi-uniformity on a set $X$ is a non empty set $\mathcal{U}$ of subsets of $X \times X$ satisfying the following properties:

(U1) If a subset $U$ of $X \times X$ contains an element of $\mathcal{U}$, then $U \in \mathcal{U}$;

(U2) The intersection of any two elements of $\mathcal{U}$ contains an element of $\mathcal{U}$;

(U3) Each element of $\mathcal{U}$ contains the diagonal of $X \times X$;

(U4) For each $U \in \mathcal{U}$, there exists $V \in \mathcal{U}$ such that $V V \subseteq U$.

A quasi-uniformity satisfying the following additional condition is called a uniformity.

(U5) For each $U \in \mathcal{U},{ }^{t} U \in \mathcal{U}$

If $\mathcal{U}$ is a quasi-uniformity on the set $X$, the elements of $\mathcal{U}$ are called entourages. Note that $X \times X$ is always an entourage. The pair $(X, \mathcal{U})$ (or the set $X$ if $\mathcal{U}$ is understood) is called a quasi-uniform space, and a uniform space if $\mathcal{U}$ is a uniformity.

Let $\mathcal{U}$ be a quasi-uniformity on a set $X$, and let $Y$ be a subset of $X$. Then the restrictions of the entourages of $\mathcal{U}$ on $Y \times Y$ form a quasi-uniformity on $Y$, called the quasi-uniformity on $Y$ induced by $\mathcal{U}$.

A basis of a quasi-uniformity $\mathcal{U}$ is a subset $\mathcal{B}$ of $\mathcal{U}$ such that each element of $\mathcal{U}$ contains an element of $\mathcal{B}$. In particular, $\mathcal{U}$ consists of all the relations on $X$ containing an element of $\mathcal{B}$. We say that $\mathcal{U}$ is generated by $\mathcal{B}$. A set $\mathcal{B}$ of subsets of $X \times X$ is a basis of some quasi-uniformity if and only if it satisfies properties (U2), (U3) and (U4).

Example 2.1 The discrete quasi-uniformity on a set $X$ (in fact a uniformity) is the quasiuniformity $\mathcal{D}$ induced by the basis consisting of the diagonal set of $X \times X$ only. In this paper, all finite sets are implicitly equipped with the discrete uniformity.

A basis of a quasi-uniformity is symmetrical if all its elements are symmetrical. The quasi-uniformity generated by a symmetrical basis is actually a uniformity. If $\mathcal{B}$ is a basis of a quasi-uniformity $\mathcal{U}$, the entourages $U^{*}$, for $U \in \mathcal{B}$, form a symmetrical basis of a uniformity $\mathcal{U}^{*}$, which is the smallest uniformity containing $\mathcal{U}$. In particular $\mathcal{U}^{*}$ is also generated by the entourages $U^{*}$, for $U \in \mathcal{U}$.

Let $(X, \mathcal{U})$ be a quasi-uniform space. The intersection $\preceq \mathcal{U}$ of the elements of $\mathcal{U}$ forms a reflexive, transitive relation on $X$, that is, a quasi-order. We also denote by $\sim \mathcal{U}$ the equivalence relation on $X$ associated with the quasi-order $\preceq \mathcal{U}$, and given by $x \sim \mathcal{U} y$ if and only if $x \preceq \mathcal{U} y$ and $y \preceq \mathcal{U} x$. If $\mathcal{U}$ is a uniformity, then $\sim_{\mathcal{U}}$ and $\preceq_{\mathcal{U}}$ coincide. 
Example 2.2 The following example will be used throughout the paper. Let $A$ be an alphabet, that is, a finite set. Let $A^{+}$and $A^{*}$ be, respectively, the free semigroup and the free monoid on $A$. The elements of $A^{*}$ are called words, and the subsets of $A^{*}$ are called languages. Let $x=a_{1} a_{2} \cdots a_{n}\left(n \geq 1, a_{i} \in A\right)$ be a word. We say that $x$ is a subword of a word $u \in A^{*}$, and we write $u \leq_{\text {sw }} x$ if $u$ can be written $u=u_{0} a_{1} u_{1} \cdots a_{n} u_{n}$ for some $u_{0}, u_{1}, \ldots, u_{n} \in A^{*}$. In particular, the empty word is a subword of each word. Let $L_{x}$ be the set of all words $u \in A^{*}$ such that $u \leq_{\mathrm{sw}} x$, and let

$$
U_{x}=\left\{(u, v) \in A^{*} \times A^{*} \mid v \leq_{\mathrm{sw}} x \Longrightarrow u \leq_{\mathrm{sw}} x\right\} .
$$

The finite intersections of entourages of the form $U_{x}\left(x \in A^{*}\right)$ form the basis of a quasiuniformity, called the subword quasi-uniformity. The associated quasi-order coincides with the subword order $\leq_{\mathrm{sw}}$ on $A^{*}$.

If $(X, \mathcal{U})$ and $(Y, \mathcal{V})$ are quasi-uniform spaces, a mapping $\varphi: X \rightarrow Y$ is said to be $\mathcal{U}$-V -uniformly continuous (or uniformly continuous if there is no ambiguity) if, for each entourage $V \in \mathcal{V}$, there exists an entourage $U \in \mathcal{U}$ such that $U \varphi \subseteq V$. In particular, such a mapping is monotonous: if $x \preceq \mathcal{U} y$, then $\varphi(x) \preceq \mathcal{V} \varphi(y)$.

For each $x \in X$, let $\mathcal{U}(x)=\{U(x) \mid U \in \mathcal{U}\}$. There exists a unique topology on $X$, called the topology induced by $\mathcal{U}$, for which $\mathcal{U}(x)$ is the filter of neighborhoods of $x$ for each $x \in X$. Note that this topology is not necessarily Hausdorff: if the pair $(x, y)$ of elements of $X$ lies in each entourage $U$, that is $x \preceq \mathcal{U} y$, then each neighborhood of $x$ contains $y$.

We implicitly assume that the set $X \times X$ is endowed with the product topology. If $\mathcal{B}$ is a symmetrical basis of a uniformity $\mathcal{U}$ and $T \subseteq X \times X$, then the topological closure of $T$ is the intersection of the $U T U$, for all entourages $U \in \mathcal{B}$ [4, TG.II.1.2, Prop. 2].

Let $(X, \mathcal{U})$ be a uniform space. A filter $\mathcal{F}$ on $X$ is a Cauchy filter if, for each entourage $U \in \mathcal{U}$, there exists $F \in \mathcal{F}$ such that $F \times F \subseteq U$. The uniform space $(X, \mathcal{U})$ is said to be complete if each Cauchy filter on $X$ is convergent. The Hausdorff completion $(\hat{X}, \hat{\mathcal{U}})$ of a uniform space $(X, \mathcal{U})$ and the uniformly continuous mapping $\imath: X \rightarrow \hat{X}$ are uniquely defined (up to isomorphism) in [4, TG.II.3.7-9] by the following universal property: every uniformly continuous mapping $\varphi$ from $(X, \mathcal{U})$ into a Hausdorff complete uniform space $\left(Y, \mathcal{U}^{\prime}\right)$ induces a unique uniformly continuous mapping $\hat{\varphi}: \hat{X} \rightarrow Y$ such that $\hat{\varphi} \circ \imath=\varphi$.

The following properties are established in [4, TG.II.3.7-9]. They will be used freely in the sequel.

Proposition 2.3 Let $(X, \mathcal{U})$ be a uniform space.

(1) The set $\imath(X)$ is dense in $\hat{X}$;

(2) Every entourage of $\mathcal{U}$ is of the form $\imath^{-1}(V)$ for some $V \in \hat{\mathcal{U}}$, and for every entourage $U \in \mathcal{U}, \imath^{-1} \imath(U)=U$

(3) If $\mathcal{B}$ is a basis of $\mathcal{U}$, then $\imath(\mathcal{B})$ is a basis of the uniformity on $\imath(X)$ induced by $\mathcal{U}^{*}$ and the closures of the entourages of $\imath(\mathcal{B})$ form a basis of $\hat{\mathcal{U}}$;

(4) the relation $\imath(x)=\imath(y)$ is the intersection of all entourages of $\mathcal{U}$.

Let us now consider a quasi-uniform space $(X, \mathcal{U})$. We define the Hausdorff completion $(\hat{X}, \hat{\mathcal{U}})$ of $(X, \mathcal{U})$ to be the Hausdorff completion of the uniform space $\left(X, \mathcal{U}^{*}\right)$. The mapping $\imath: X \rightarrow \hat{X}$ is $\mathcal{U}^{*}-\hat{\mathcal{U}}$-uniformly continuous, but it is not $\mathcal{U}-\hat{\mathcal{U}}$-uniformly continuous in general. We also denote by $\imath$ the induced mapping from $X \times X$ into $\hat{X} \times \hat{X}$. 
The equivalence relation induced by $\imath$ on $X$ is equal to

$$
\bigcap_{V \in \mathcal{U}^{*}} V=\bigcap_{U \in \mathcal{U}} U^{*}=\bigcap_{U \in \mathcal{U}} U \cap \bigcap_{U \in \mathcal{U}}{ }^{t} U=\preceq \mathcal{U} \cap^{t} \preceq \mathcal{U}=\sim_{\mathcal{U}} .
$$

Thus $\imath(\preceq \mathcal{U})$ is an order relation on $\imath(X)$ and $(\imath(X), \imath(\preceq \mathcal{U}))$ is the poset naturally associated with the quasi-ordered set $(X, \preceq \mathcal{U})$.

\subsection{Quasi-écarts}

It is well known that certain topologies can be defined by distance functions. Similarly, certain quasi-uniformities can be defined by quasi-écarts, a generalization of distance functions. The formal definition of quasi-écarts is as follows.

A quasi-écart on a set $X$ is a mapping $e: X \times X \rightarrow[0,+\infty]$ satisfying the following conditions:

(E1) For each $x \in X, e(x, x)=0$;

(E2) If $x, y, z \in X$, then $e(x, z) \leq e(x, y)+e(y, z)$.

We say that the quasi-écart $e$ is ultrametric if it satisfies the stronger condition:

$\left(\mathrm{E}^{\prime} 2\right) e(x, z) \leq \max (e(x, y), e(y, z))$ for all $x, y, z \in X$.

An écart is a quasi-écart $e$ satisfying also

(E3) $e(x, y)=e(y, x)$ for all $x, y \in X$.

A quasi-écart $e$ on a set $X$ naturally defines a quasi-uniformity $\mathcal{U}$ on $X$. A basis of $\mathcal{U}$ is given by the subsets of $X \times X$ of the form

$$
U_{\varepsilon}=e^{-1}([0, \varepsilon[)=\{(x, y) \in X \times X \mid e(x, y)<\varepsilon\} \quad(\varepsilon>0)
$$

If $e$ is an écart, then $\mathcal{U}$ is a uniformity. Two quasi-écarts $e$ and $f$ on the set $X$ are said to be uniformly equivalent if they define the same quasi-uniformity.

It is easily verified that if $e$ and $f$ are quasi-écarts, respectively on $X$ and on $Y$, and if $\mathcal{U}$ and $\mathcal{V}$ are the quasi-uniformities defined by these quasi-écarts, then a mapping $\varphi: X \rightarrow Y$ is $\mathcal{U}$ - $\mathcal{V}$-uniformly continuous if and only if

$$
\forall \varepsilon>0, \exists \alpha>0, \forall x, x^{\prime} \in X, \quad e\left(x, x^{\prime}\right)<\alpha \Longrightarrow f\left(\varphi(x), \varphi\left(x^{\prime}\right)\right)<\varepsilon .
$$

Not all quasi-uniformities can be defined by a quasi-écart. Those quasi-uniformities which can be thus defined are characterized by the following property (a simple variant of [4, TG.IX.1, Prop. 2]).

Proposition 2.4 Let $\mathcal{U}$ be a quasi-uniformity. Then $\mathcal{U}$ can be defined by a quasi-écart if and only if $\mathcal{U}$ has a countable basis.

\section{Totally bounded transitive quasi-uniformities}

As explained in the introduction, we are eventually interested in profinite completions of the free semigroup, that is, in completions which are compact and totally disconnected. This translates in terms of uniformities as follows. 
A quasi-uniformity $\mathcal{U}$ on $X$ is said to be totally bounded if, for each entourage $U$, there exist finitely many subsets $B_{1}, \ldots, B_{n}$ of $X$ such that $X=\bigcup_{i} B_{i}$ and $\bigcup_{i}\left(B_{i} \times B_{i}\right) \subseteq U$. As we will see below, the property of $\mathcal{U}$ to be totally bounded is linked with the compactness of $\hat{X}$, and therefore it is an analogue of precompactness. This analogy is made precise as follows. We say that a quasi-écart $e$ on a set $X$ is precompact if, for each $\varepsilon>0$, there exist finitely many elements of $X$, say $x_{1}, \ldots, x_{n}$, such that

for each $x \in X$, there exists $1 \leq i \leq n$ such that $\max \left(e\left(x, x_{i}\right), e\left(x_{i}, x\right)\right)<\varepsilon$.

It is easy to verify that $e$ is precompact if and only if the quasi-uniformity defined by $e$ on $X$ is totally bounded. It is therefore not surprising that we have the following characterization of totally bounded quasi-uniformities.

Proposition 3.1 Let $(X, \mathcal{U})$ be a quasi-uniform space.

(1) $\mathcal{U}$ is totally bounded if and only if $\mathcal{U}^{*}$ is totally bounded.

(2) $\hat{X}$ is compact if and only if $\mathcal{U}$ is totally bounded.

Proof. The first statement is immediate by definition of $\mathcal{U}^{*}$. The second one then follows from [4, TG.II.29, Thm. 3].

Example 3.2 Let $\mathcal{U}$ be the subword quasi-uniformity (see Example 2.2). For each $x \in$ $A^{+}$, we have

$$
U_{x}=A^{*} \times\left(A^{*} \backslash L_{x}\right) \cup L_{x} \times A^{*},
$$

so $\mathcal{U}$ is totally bounded.

The quasi-uniformity $\mathcal{U}$ on $X$ is said to be transitive if it has a basis consisting of transitive entourages. Again, this property is analogous to a well known metric property: it is easily verified that if $e$ is an ultrametric quasi-écart, then the entourages of the form $\{(x, y) \in X \times X \mid e(x, y)<\varepsilon\}(\varepsilon>0)$ are transitive. Therefore the quasi-uniformity defined by an ultrametric quasi-écart is transitive.

Example 3.3 The entourages $U_{x}\left(x \in A^{+}\right)$are easily seen to be transitive (Examples 2.2 and 3.2 ), so the subword quasi-uniformity is transitive.

In the sequel, $\bar{T}$ denotes the topological closure of a subset $T$ of $\hat{X}$ or $\hat{X} \times \hat{X}$, in the topology induced by $\hat{\mathcal{U}}$.

Proposition 3.4 Let $(X, \mathcal{U})$ be a quasi-uniform space. If $\mathcal{U}$ is transitive, then so are $\mathcal{U}^{*}$ and $\hat{\mathcal{U}}$.

Proof. Since $\mathcal{U}$ is transitive, it admits a transitive basis $\mathcal{B}$. Now a basis of $\mathcal{U}^{*}$ is formed by the $U^{*}$, for $U \in \mathcal{B}$. But the transitivity of $U$ immediately implies that of $U^{*}$, so $\mathcal{U}^{*}$ is transitive.

To prove that $\hat{\mathcal{U}}$ is transitive, it suffices to show that $\overline{\imath\left(U^{*}\right)}$ is transitive for each transitive entourage $U \in \mathcal{U}$. Now we have

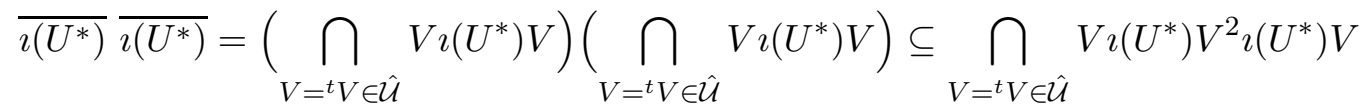


For each symmetrical entourage $W \in \hat{\mathcal{U}}$, let $V \in \hat{\mathcal{U}}$ be a symmetrical entourage such that $V^{2} \subseteq \overline{\imath\left(U^{*}\right)} \cap W$. Then

$$
\overline{\imath\left(U^{*}\right)} \overline{\imath\left(U^{*}\right)} \subseteq V \imath\left(U^{*}\right) V^{2} \imath\left(U^{*}\right) V \subseteq W \imath\left(U^{*} \overline{\imath\left(U^{*}\right)} \imath\left(U^{*}\right) W .\right.
$$

Now let us consider an element of $\imath\left(U^{*}\right) \overline{\imath\left(U^{*}\right)} \imath\left(U^{*}\right)$, say $(\imath(x), \imath(y))$ with $x, y \in X$. Then there exist $z, t \in \hat{X}$ such that $(\imath(x), z) \in \imath\left(U^{*}\right),(z, t) \in \overline{\imath\left(U^{*}\right)}$ and $(t, \imath(y)) \in \imath\left(U^{*}\right)$. It follows that $z=\imath\left(z^{\prime}\right)$ and $t=\imath\left(t^{\prime}\right)$ for some $z^{\prime}, t^{\prime} \in X$, such that $\left(x, z^{\prime}\right),\left(t^{\prime}, y\right) \in U^{*}=$ $\imath^{-1} \imath\left(U^{*}\right)$. Moreover,

$$
\left(z^{\prime}, t^{\prime}\right) \in \imath^{-1}\left(\overline{\imath\left(U^{*}\right)} \cap(\imath(X) \times \imath(X))\right)=\imath^{-1} \imath\left(U^{*}\right)=U^{*} .
$$

But $U$ is transitive, and hence so is $U^{*}$. Therefore $(x, y) \in U^{*}$ and $(\imath(x), \imath(y)) \in \imath\left(U^{*}\right)$. Thus

$$
\imath\left(U^{*}\right) \overline{\imath\left(U^{*}\right)} \imath\left(U^{*}\right)=\imath\left(U^{*}\right)
$$

and hence $\overline{\imath\left(U^{*}\right)}$ is transitive.

We now assume that $\mathcal{U}$ is a totally bounded, transitive quasi-uniformity. Let $U$ be a transitive entourage. There exists a finite cover of $X, X=B_{1} \cup \cdots \cup B_{n}$, such that $\bigcup_{i=1}^{n}\left(B_{i} \times B_{i}\right) \subseteq U$. If $B_{i} \times B_{j}$ meets $U$, then $B_{i} \times B_{j} \subseteq U$ by transitivity. In particular, if both $B_{i} \times B_{j}$ and $B_{j} \times B_{i}(1 \leq i, j \leq n)$ have a non empty intersection with $U$ - for instance if $B_{i} \cap B_{j} \neq \emptyset-$, then $\left(B_{i} \cup B_{j}\right) \times\left(B_{i} \cup B_{j}\right) \subseteq U$. So we may assume that the cover $\left(B_{i}\right)_{i}$ is a partition of $X$, and that for all $i, j$, either $B_{i} \times B_{j}$ or $B_{j} \times B_{i}$ does not meet $U$. This uniquely determines the $B_{i}$, which are called $U$-blocks. The $U$-blocks are ordered by the relation $B_{i} \preceq B_{j}$ if $B_{i} \times B_{j} \subseteq U$. Thus

$$
U=\bigcup_{B_{i} \preceq B_{j}} B_{i} \times B_{j} .
$$

Observe that if $x \in B_{i}, y \in B_{j}(1 \leq i, j \leq n)$ and $x \preceq \mathcal{U} y$, then $(x, y) \in U$, so $B_{i} \times B_{j}$ meets $U$ and hence $i \preceq j$. Therefore the union of a set $C$ of $U$-blocks is a $\preceq \mathcal{U}$-order ideal if and only if $C$ is a $\preceq$-order ideal.

The subsets of $X$ which arise as $U$-blocks for some transitive entourage $U \in \mathcal{U}$, are called $\mathcal{U}$-blocks. Observe that a finite family of $\mathcal{U}$-blocks is in fact a family of $U$-blocks for some $U \in \mathcal{U}$, since a finite intersection of entourages of $\mathcal{U}$ is an entourage.

Example 3.5 We have seen that the subword quasi-uniformity $\mathcal{U}$ is totally bounded and transitive (Examples 3.2 and 3.3). If $x \in A^{+}$, the formula given in Example 3.2 shows that the $U_{x}$-blocks are $L_{x}$ and $A^{*} \backslash L_{x}$. The $\mathcal{U}$-blocks are the Boolean combinations of sets of the form $L_{x}$.

If $U \in \mathcal{U}$ is transitive, then the entourage $U^{*}$ of $\mathcal{U}^{*}$ is transitive as well. If $X=$ $B_{1} \cup \cdots \cup B_{n}$ is the partition of $X$ in $U$-blocks, then it is immediately verified that $U^{*}=\left(B_{1} \times B_{1}\right) \cup \cdots \cup\left(B_{n} \times B_{n}\right)$, so the $U$-blocks are also the $U^{*}$-blocks. As a result, the $\mathcal{U}$-blocks are the $\mathcal{U}^{*}$-blocks.

Proposition 3.6 Let $\mathcal{U}$ be a totally bounded, transitive quasi-uniformity on the set $X$. The $\mathcal{U}$-blocks form a basis of clopen subsets of the topology induced by $\mathcal{U}^{*}$. 
Proof. Let $U$ be a transitive entourage of $\mathcal{U}$ and let $X=B_{1} \cup \cdots \cup B_{n}$ be the partition of $X$ in $U$-blocks. Then $U^{*}=\bigcup_{i=1}^{n}\left(B_{i} \times B_{i}\right)$. In particular, $U^{*}(x)=B_{i}$ for each $x \in B_{i}$, so $B_{i}$ is a neighborhood of each of its points, and hence $B_{i}$ is open. Since the complement of $B_{i}$ is the (finite) union of the other $U$-blocks, it follows that $B_{i}$ is closed as well.

Let $G$ be a non empty open set for the topology induced by $\mathcal{U}^{*}$ and let $x \in G$. There exists a transitive entourage $U \in \mathcal{U}$ such that $U^{*}(x) \subseteq G$. But $U^{*}(x)$ is one of the $U$-blocks, so each non empty open set contains a $\mathcal{U}$-block.

Proposition 3.7 Let $\mathcal{U}$ be a totally bounded, transitive quasi-uniformity on the set $X$. If $B$ is a $\mathcal{U}$-block, then $\overline{\imath(B)}$ is a $\hat{\mathcal{U}}$-block. Moreover, $\imath^{-1}(\overline{\imath(B)})=B$.

Proof. Let $U \in \mathcal{U}$ be a transitive entourage and let $X=B_{1} \cup \cdots \cup B_{n}$ be the partition of $X$ in $U$-blocks. Since $U^{*}=\left(B_{1} \times B_{1}\right) \cup \cdots \cup\left(B_{n} \times B_{n}\right)$, we have

$$
\begin{aligned}
\overline{\imath\left(U^{*}\right)} & \left.=\overline{\left(\overline{\left(B_{1}\right)}\right.} \times \overline{\imath\left(B_{1}\right)} \cup \cup \cup \overline{\left(\overline{\imath\left(B_{n}\right)}\right.} \times \overline{\imath\left(B_{n}\right)}\right) \\
\hat{X} & =\overline{\imath(X)}=\overline{\imath\left(B_{1}\right)} \cup \cdots \cup \overline{\imath\left(B_{n}\right)} .
\end{aligned}
$$

Now the proof of Proposition 3.4 shows that $\overline{\imath\left(U^{*}\right)}$ is transitive. If $\overline{\imath\left(B_{i}\right)}$ and $\overline{\imath\left(B_{j}\right)}(i \neq j)$

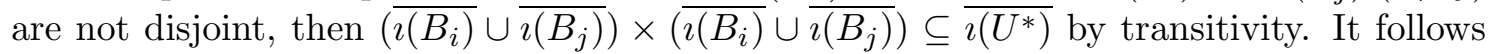
that

$$
\left(B_{i} \cup B_{j}\right) \times\left(B_{i} \cup B_{j}\right) \subseteq \imath^{-1}\left(\overline{\imath\left(U^{*}\right)} \cap(\imath(X) \times \imath(X))\right)=U^{*},
$$

a contradiction. So the $\overline{\imath\left(B_{i}\right)}$ are pairwise disjoint, and hence they are the $\overline{\imath\left(U^{*}\right)}$-blocks.

The equality $\imath^{-1}(\overline{\imath(B)})=B$ follows from the fact that $\imath^{-1}\left(\overline{\imath\left(U^{*}\right)}\right)=U^{*}$.

\section{Semigroups}

We now turn our attention to quasi-uniform semigroups, that is, semigroups which are equipped with a quasi-uniformity for which the multiplication is uniformly continuous. Before we can state and prove the properties of such semigroups which are relevant to our purpose (Section 4.3), we need to recall certain properties of ordered semigroups and of compact semigroups.

\subsection{Syntactic properties}

An ordered semigroup is a pair $(S, \leq)$, where $S$ is a semigroup, and $\leq$ is an order relation on $S$ such that $s \leq s^{\prime}$ and $t \leq t^{\prime}\left(s, s^{\prime}, t, t^{\prime} \in S\right)$ implies $s t \leq s^{\prime} t^{\prime}$. We denote by $S^{1}$ the monoid equal to $S$, if $S$ has an identity element, and to $S \cup\{1\}$ otherwise. Let $P$ be an order ideal of $(S, \leq)$. The syntactic quasi-order $\preceq_{P}$ on $S$ is given by

$$
s \preceq_{P} t \quad \text { if and only if } \quad x t y \in P \Rightarrow x s y \in P \text { for all } x, y \in S^{1} .
$$

Let $\sim_{P}$ be the induced congruence on $S$. Let $\mu: S \rightarrow S(P)$ be the projection onto the quotient semigroup $S(P)=S / \sim_{P}$. Then $S(P)$ is naturally ordered by

$$
\mu(s) \leq_{P} \mu(t) \quad \text { if and only if } \quad s \preceq_{P} t .
$$

The ordered semigroup $\left(S(P), \leq_{P}\right)$ is called the syntactic ordered semigroup of $P$, and $\mu$ is the syntactic morphism of $P$. It is well known [13] that $\mu$ is a monotonous morphism, that $P=\mu^{-1} \mu(P)$, and that each onto monotonous morphism $\varphi: S \rightarrow T$ such that $P=$ 
$\varphi^{-1} \varphi(P)$ (we say that $\varphi$ recognizes $P$ ) factorizes through $\mu$. In particular, $S(P)$ is finite if and only if there exists a finite ordered semigroup $(T, \leq)$ and a monotonous morphism $\varphi: S \rightarrow T$ recognizing $P$. If this is the case, we say that $P$ is recognizable.

For any semigroup $S,(S,=)$ is an ordered semigroup. The above results and definitions can be simplified in that case, by dropping all mentions of order for semigroups and monotonicity for morphisms. We will freely talk about recognizable subsets of (unordered) semigroups, thus meaning recognizable subsets of the semigroup equipped with the trivial order. In fact, the recognizable subsets of $(S, \leq)$ are exactly the recognizable subsets of $(S,=)$ which are $\leq$-order ideals.

\subsection{Compact semigroups}

If a semigroup $S$ is endowed with a topology such that the multiplication in $S$ is continuous, we say that $S$ is a topological semigroup. If $S$ is compact for this topology, we say that $S$ is a compact semigroup. The following result is known as Hunter's Lemma [10, Lemma 4].

Proposition 4.1 Let $S$ be a compact semigroup and let $P$ be a clopen subset of $S$. Then the syntactic congruence of $P$ is clopen.

This yields the following corollary.

Corollary 4.2 Let $S$ be a compact semigroup and let $P$ be a clopen subset of $S$. Then $P$ is recognizable and its syntactic morphism is uniformly continuous.

Proof. Let $\mu: S \rightarrow S(P)$ be the syntactic morphism of $P$, and let $\sim_{P}$ be its syntactic congruence. By Proposition 4.1, $\sim_{P}$ is clopen. So for each $s \in S$, there exists an open neighborhood $G$ of $s$ such that $G \times G \subseteq \sim_{P}$. Therefore $G$ is contained in the $\sim_{P}$-class of $s$. This proves that the $\sim_{P}$-classes form an open partition of $S$. By compactness, this partition is finite, and hence $S(P)$ is finite, that is, $P$ is recognizable. In addition, for each

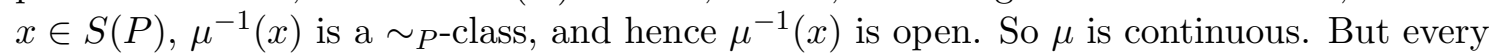
compact space admits a unique uniformity compatible with its topology [4, TG.II.4.1, Thm. 1], and a continuous function on a compact space is always uniformly continuous [4, TG.II.4.1, Thm. 2], so the corollary is proved.

\subsection{Quasi-uniform semigroups}

Let now $S$ be a semigroup and let $\mathcal{U}$ be a quasi-uniformity (resp. uniformity) on $S$. We say that $(S, \mathcal{U})$ is a quasi-uniform (resp. uniform) semigroup if the multiplication in $S$ is uniformly continuous.

Example 4.3 The free monoid $A^{*}$, equipped with the subword quasi-uniformity (see Example 2.2) is a quasi-uniform semigroup.

Indeed, for each $x \in A^{*}$, let $P(x)$ be the set of prefixes of $x$, that is, the set of elements $y \in A^{*}$ such that $x=y z$ for some $z \in A^{*}$. Similarly, let $S(x)$ be the set of suffixes of $x$, that is, the set of elements $z \in A^{*}$ such that $x=y z$ for some $y \in A^{*}$. Both $P(x)$ and $S(x)$ are finite sets. One verifies that if $(u, v) \in \bigcap_{y \in P(x)} U_{y}$ and $\left(u^{\prime}, v^{\prime}\right) \in \bigcap_{z \in P(x)} U_{z}$, then $\left(u u^{\prime}, v v^{\prime}\right) \in U_{x}$. So the multiplication in $A^{*}$ is uniformly continuous with respect to the subword quasi-uniformity. 
Let $(S, \mathcal{U})$ be a quasi-uniform semigroup. It is immediately verified that the multiplication of $S$ is also uniformly continuous with respect to $\mathcal{U}^{*}$, and it follows that $\hat{S}$ can be endowed with a multiplication which makes $(\hat{S}, \hat{\mathcal{U}})$ a uniform, complete semigroup, and which makes $\imath$ a $\mathcal{U}^{*}-\hat{\mathcal{U}}$-uniformly continuous morphism.

Corollary 4.2 translates as follows for quasi-uniform semigroups.

Proposition 4.4 Let $(S, \mathcal{U})$ be a quasi-uniform semigroup such that $\mathcal{U}$ is totally bounded. If $P$ is a clopen subset of $\hat{S}$, then $\imath^{-1}(P)$ is recognizable and its syntactic morphism is uniformly continuous with respect to $\mathcal{U}^{*}$.

Proof. Since $\mathcal{U}$ is totally bounded, $\hat{S}$ is a compact semigroup. By Corollary $4.2, P$ is a recognizable subset of $\hat{S}$, and its syntactic morphism $\mu: \hat{S} \rightarrow S(P)$ is uniformly continuous. Now the morphism $\eta \circ \imath: S \rightarrow S(P)$ recognizes $\imath^{-1}(P)$, so $\imath^{-1}(P)$ is recognizable and its syntactic morphism factorizes through $\eta \circ \imath$. The proposition follows from the fact that $\imath$ is $\mathcal{U}^{*}-\hat{\mathcal{U}}$-uniformly continuous.

That implies that, if the quasi-uniformity $\mathcal{U}$ is totally bounded and transitive, then the $\mathcal{U}$-blocks are recognizable.

Example 4.5 Let us consider again the subword quasi-uniformity of Example 2.2. For each $x \in A^{*}$, the entourage $U_{x}$ is exactly the syntactic quasi-order of the language $L_{x}$.

Corollary 4.6 Let $(S, \mathcal{U})$ be a quasi-uniform semigroup such that $\mathcal{U}$ is totally bounded and transitive. The $\mathcal{U}$-blocks are recognizable, and their syntactic morphisms are uniformly continuous with respect to $\mathcal{U}^{*}$.

Proof. By Proposition 3.7, each $\mathcal{U}$-block $B$ satisfies $B=\imath^{-1}(\overline{\imath(B)})$ and $\overline{\imath(B)}$ is a $\hat{\mathcal{U}}$-block. By Proposition 3.6, $\overline{\imath(B)}$ is clopen: it now suffices to apply Proposition 4.4.

One can verify easily that the syntactic morphism of a recognizable subset $P$ of $S$ is uniformly continuous with respect to $\mathcal{U}$ itself if $P$ is a $\preceq \mathcal{U}$ order ideal.

Finally, Corollary 4.6 has the following important consequence.

Proposition 4.7 Let $(S, \mathcal{U})$ be a quasi-uniform semigroup such that $S$ is finitely generated and $\mathcal{U}$ is totally bounded and transitive. Then $\mathcal{U}$ admits a countable basis.

Proof. We prove in fact that $\mathcal{U}$ admits only countably many transitive entourages. Indeed, for each such entourage $U$, let $S=B_{1} \cup \ldots \cup B_{n}$ be the partition of $S$ in $U$-blocks and let $\preceq$ the associated order relation on the set of $U$-blocks. Then $U=\bigcup_{B_{i} \prec B_{j}} B_{i} \times B_{j}$.

So for a given choice of a partition of $S$ as a union of $\mathcal{U}$-blocks, $S=B_{1} \cup \ldots \cup B_{n}$, there are finitely many entourages $U$ such that the $B_{i}$ are the $U$-blocks. Thus it suffices to prove that there are only countably many such partitions. But each $\mathcal{U}$-block is a recognizable subset of $S$ by Corollary 4.6. So it suffices to show that $S$ has only countably many recognizable subsets. Now a recognizable subset $P$ of $S$ is entirely specified by a morphism $\varphi: S \rightarrow T$ from $S$ into a finite semigroup $T$ and by the subset $\varphi(P)$ of $T$.

Now $S$ is finitely generated, so there are only countably many morphisms from $S$ into finite semigroups, and this concludes the proof.

By Proposition 2.4, it follows that if $(S, \mathcal{U})$ is a quasi-uniform semigroup such that $S$ is finitely generated and $\mathcal{U}$ is totally bounded and transitive, then $\mathcal{U}$ can be defined by an ultrametric quasi-écart. We can adapt the proof of [9, Theorem 6.8] to show the following, more precise result. We will give an even more precise result in Section 5.3. 
Corollary 4.8 Let $(S, \mathcal{U})$ be a quasi-uniform semigroup such that $S$ is finitely generated and $\mathcal{U}$ is totally bounded and transitive. Then $\mathcal{U}$ can be defined by a precompact ultrametric quasi-écart.

\section{Varieties}

We now consider quasi-uniformities on finitely generated free semigroups. We will show that certain classes of such quasi-uniformities can be characterized algebraically. First we need some definitions.

\subsection{Definitions}

A pseudovariety of ordered semigroups is a class of finite ordered semigroups which is closed under taking (ordered) subsemigroups, (ordered) finite direct products and monotonous homomorphic images. Similarly, a pseudovariety of semigroups is a class of finite semigroups which is closed under taking subsemigroups, finite direct products and homomorphic images. Observe that pseudovarieties of semigroups are special cases of pseudovarieties of ordered semigroups. More precisely, if $\mathbf{V}$ is a pseudovariety of semigroups, then the class of all ordered semigroups of the form $(S, \leq)$, where $S \in \mathbf{V}$, is a pseudovariety of ordered semigroups. It is generated by the ordered semigroups $(S,=)$ where $S \in \mathbf{V}$. We will use the following property [13].

Lemma 5.1 Let $\mathbf{V}$ be a pseudovariety of ordered semigroups. Then $\mathbf{V}$ is generated by the syntactic ordered semigroups it contains. More precisely, if $\sigma: A^{+} \rightarrow(S, \leq)$ is a morphism onto an ordered semigroup in $\mathbf{V}$, there exist finitely many languages $L_{1}, \ldots, L_{n}$ in $A^{+}$such that the syntactic ordered semigroup of each $L_{i}$ is a monotonous homomorphic image of $(S, \leq)$, and such that $(S, \leq)$ embeds in the direct product $\left(S\left(L_{1}\right), \leq_{L_{1}}\right) \times \cdots \times\left(S\left(L_{n}\right), \leq_{L_{n}}\right)$.

A variety of quasi-uniformities is a family $\mathcal{U}=\left(\mathcal{U}_{A}\right)_{A}$ of totally bounded, transitive quasi-uniformities defined on each free semigroup $A^{+}$( $A$ finite), such that each $\left(A^{+}, \mathcal{U}_{A}\right)$ is a quasi-uniform semigroup, and such that the morphisms between free semigroups are uniformly continuous.

By Corollary 4.8, each quasi-uniformity arising in a variety of quasi-uniformities can be described by an ultrametric quasi-écart. We will return to this idea in Section 5.3.

Now we define two correspondences, from varieties of quasi-uniformities to pseudovarieties of ordered semigroups, and vice versa.

If $\mathcal{U}$ is a variety of quasi-uniformities, we let $\mathbf{V}(\mathcal{U})$ be the pseudovariety of ordered semigroups generated by the ordered syntactic semigroups of the finite unions of $\mathcal{U}_{A}$-blocks which constitute $\preceq \mathcal{U}_{A}$-order ideals (see Corollary 4.6).

Conversely, let $\mathbf{V}$ be a pseudovariety of ordered semigroups. For each morphism $\varphi: A^{+} \rightarrow S$ from a free semigroup ( $A$ finite) into an ordered semigroup $(S, \leq)$ in $\mathbf{V}$, we let

$$
U_{A, \varphi}=\left\{(u, v) \in A^{+} \times A^{+} \mid \varphi(u) \leq \varphi(v)\right\} .
$$

Observe that $U_{A, \varphi}$ is transitive, and that $U_{A, \varphi}^{*}=\bigcup_{s \in S}\left(\varphi^{-1}(s) \times \varphi^{-1}(s)\right)$. In fact, the $\varphi^{-1}(s)(s \in S)$ are the $U_{A, \varphi}$-blocks.

When $A$ is fixed and $\varphi$ runs over all morphisms from $A^{+}$into an element of $\mathbf{V}$, the $U_{A, \varphi}$ form the basis of a totally bounded, transitive quasi-uniformity on $A^{+}$, denoted by $\mathcal{U}(\mathbf{V})_{A}$. Let $\mathcal{U}(\mathbf{V})=\left(\mathcal{U}(\mathbf{V})_{A}\right)_{A}$. 
Lemma 5.2 Let $\mathbf{V}$ be a pseudovariety of ordered finite semigroups. Then $\mathcal{U}(\mathbf{V})$ is a variety of quasi-uniformities.

Proof. Let $A$ be an alphabet and let $\varphi: A^{+} \rightarrow S$ be a morphism into an element $(S, \leq)$ of $\mathbf{V}$. If $(u, v),\left(u^{\prime}, v^{\prime}\right) \in U_{A, \varphi}$, then $\varphi(u) \leq \varphi(v)$ and $\varphi\left(u^{\prime}\right) \leq \varphi\left(v^{\prime}\right)$. Therefore $\varphi\left(u u^{\prime}\right) \leq$ $\varphi\left(v v^{\prime}\right)$, that is, $\left(u u^{\prime}, v v^{\prime}\right) \in U_{A, \varphi}$. So $\left(A^{+}, \mathcal{U}_{A}\right)$ is a quasi-uniform semigroup.

Similarly, one verifies that if $\alpha: A^{+} \rightarrow B^{+}$is a semigroup, then $\left(U_{A, \varphi \circ \alpha}\right) \alpha \subseteq U_{B, \varphi}$ for each morphism $\varphi: B^{+} \rightarrow S$, so that $\alpha$ is uniformly continuous.

Given an alphabet $A$, it will be useful to have a characterization of the quasi-uniformity $\mathcal{U}(\mathbf{V})_{A}$, in terms of the languages of $A^{+}$recognized by the elements of $\mathbf{V}$.

Lemma 5.3 Let $\mathbf{V}$ be a pseudovariety of ordered finite semigroups and let $A$ be an alphabet. Then the finite intersections of syntactic quasi-orders of languages recognized by ordered semigroups in $\mathbf{V}$, form a basis of $\mathcal{U}(\mathbf{V})_{A}$.

Proof. Let $\varphi: A^{+} \rightarrow(S, \leq)$ be a morphism onto an element of $\mathbf{V}$. In view of Lemma 5.1, there exist finitely many languages $L_{1}, \ldots, L_{n} \subseteq A^{+}$, with syntactic morphisms respectively $\mu_{1}: A^{+} \rightarrow\left(S\left(L_{1}\right), \leq L_{1}\right), \ldots, \mu_{n}: A^{+} \rightarrow\left(S\left(L_{n}\right), \leq L_{n}\right)$ such that the $\left(S\left(L_{i}\right), \leq_{L_{i}}\right)$ lie in $\mathbf{V}$, and such that $U_{A, \varphi}$ is the intersection of the $U_{A, \mu_{i}}$. To conclude, we observe that $U_{A, \mu_{i}}$ is exactly the syntactic quasi-order of $L_{i}$.

Let us also notice the following characterization of membership in a pseudovariety of ordered monoids $\mathbf{V}$, in terms of the variety of quasi-uniformities $\mathcal{U}(\mathbf{V})$.

Proposition 5.4 Let $\mathbf{V}$ be a pseudovariety of ordered semigroups and let $(T, \leq)$ be a finite ordered semigroup. Let $A$ be an alphabet and let $\tau: A^{+} \rightarrow T$ be an onto semigroup morphism. Then $(T, \leq) \in \mathbf{V}$ if and only if there exists an entourage $U \in \mathcal{U}(\mathbf{V})_{A}$ such that $\tau(u) \leq \tau(v)$ for all $(u, v) \in U$.

Proof. If $(T, \leq) \in \mathbf{V}$, then $\tau(u) \leq \tau(v)$ for all $(u, v) \in U_{A, \tau}$, and $U_{A, \tau} \in \mathcal{U}(\mathbf{V})_{A}$. Conversely, let us assume that there exists $U \in \mathcal{U}(\mathbf{V})_{A}$ such that $\tau(u) \leq \tau(v)$ for all $(u, v) \in U$. By definition of $\mathcal{U}(\mathbf{V})_{A}$, we may assume that $U=U_{A, \varphi}$ for some onto morphism $\varphi: A^{+} \rightarrow S$ with $(S, \leq) \in \mathbf{V}$. This means that

$$
\varphi(u) \leq \varphi(v) \quad \Longrightarrow \quad \tau(u) \leq \tau(v) \quad \text { for all } u, v \in A^{+}
$$

and hence there exists an onto monotonous morphism $\sigma: S \rightarrow T$. It follows immediately that $(T, \leq) \in \mathbf{V}$.

\subsection{Main theorem}

We show that the two correspondences above are mutually reciprocal and monotonous, an analogue of Eilenberg's variety theorem $[8,12,13]$.

Theorem 5.5 Let $\mathbf{V}$ and $\mathbf{V}^{\prime}$ be pseudovarieties of ordered semigroups, and let $\mathcal{U}$ and $\mathcal{U}^{\prime}$ be varieties of quasi-uniformities.

(1) If $\mathbf{V} \subseteq \mathbf{V}^{\prime}$, then $\mathcal{U}(\mathbf{V})$ is less fine than $\mathcal{U}\left(\mathbf{V}^{\prime}\right)$, that is, for each alphabet $A, \mathcal{U}(\mathbf{V})_{A} \subseteq$ $\mathcal{U}\left(\mathbf{V}^{\prime}\right)_{A}$.

(2) If $\mathcal{U}_{A} \subseteq \mathcal{U}_{A}^{\prime}$ for each alphabet $A$, then $\mathbf{V}(\mathcal{U}) \subseteq \mathbf{V}\left(\mathcal{U}^{\prime}\right)$. 
Proof. By definition, for each alphabet $A$, a basis of $\mathcal{U}(\mathbf{V})_{A}$ is given by the $U_{A, \varphi}$, where $\varphi$ runs over all morphisms from $A^{+}$into an ordered semigroup $(S, \leq) \in \mathbf{V}$. So if $\mathbf{V} \subseteq \mathbf{V}^{\prime}$, then the entourages of $\mathcal{U}(\mathbf{V})_{A}$ are also entourages of $\mathcal{U}\left(\mathbf{V}^{\prime}\right)_{A}$. This proves the first assertion.

Let us now verify the second assertion. By definition, $\mathbf{V}(\mathcal{U})$ is generated by the ordered syntactic semigroups of the finite unions of $\mathcal{U}_{A}$-blocks which constitute $\preceq \mathcal{U}_{A}$-order ideals, and we are assuming that each element of $\mathcal{U}_{A}$ is also an element of $\mathcal{U}_{A}^{\prime}$. It follows from this assumption that each $\mathcal{U}_{A}$-block is a $\mathcal{U}_{A}^{\prime}$-block, and also that the quasi-order $\preceq \mathcal{U}_{A}$ contains $\preceq_{\mathcal{U}_{A}^{\prime}}$. In particular, each $\preceq_{\mathcal{U}_{A}}$-order ideal is a $\preceq_{\mathcal{U}_{A}^{\prime}}$-order ideal. Thus the generators of $\mathbf{V}(\mathcal{U})$ are elements of $\mathbf{V}\left(\mathcal{U}^{\prime}\right)$, and $\mathbf{V}(\mathcal{U}) \subseteq \mathbf{V}\left(\mathcal{U}^{\prime}\right)$.

To lighten the notation, we denote by $\preceq \mathbf{v}$ the quasi-order $\preceq \mathcal{U}(\mathbf{V})_{A}$ on $A^{+}$.

Corollary 5.6 The correspondences $\mathbf{V} \mapsto \mathcal{U}(\mathbf{V})$ and $\mathcal{U} \mapsto \mathbf{V}(\mathcal{U})$, between pseudovarieties of ordered semigroups and varieties of quasi-uniformities, are mutually reciprocal. That is, we have

$$
\mathcal{U}(\mathbf{V}(\mathcal{U}))=\mathcal{U} \quad \text { and } \quad \mathbf{V}(\mathcal{U}(\mathbf{V}))=\mathbf{V}
$$

for each variety of quasi-uniformities $\mathcal{U}$ and for each pseudovariety of ordered semigroups V.

Proof. It follows immediately from Theorem 5.5 that the two correspondences are oneto-one. Now we verify that for each pseudovariety of finite ordered semigroups $\mathbf{V}$, we have $\mathbf{V}(\mathcal{U}(\mathbf{V}))=\mathbf{V}$.

Let $\mathbf{W}=\mathbf{V}(\mathcal{U}(\mathbf{V}))$. Then $\mathbf{W}$ is generated by the syntactic ordered semigroups of

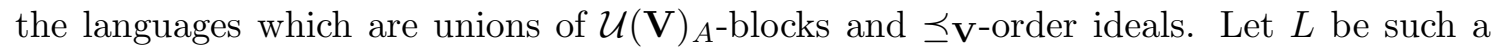
language. Then there exists an entourage $U$ in $\mathcal{U}(\mathbf{V})_{A}$ such that $L$ is a union of $U$-blocks. By definition of $\mathcal{U}(\mathbf{V})_{A}$, we can assume that $U=U_{A, \varphi}$ where $\varphi: A^{+} \rightarrow(S, \leq)$ where $(S, \leq) \in \mathbf{V}$. Since $\preceq_{\mathcal{U}}$ is contained in $U$, if $u, v \in A^{+}$and $u \preceq_{\mathcal{U}} v$, then $\varphi(u) \leq \varphi(v)$. So $\varphi(L)$ is an order ideal of $(S, \leq)$, and $\varphi$ recognizes $L$. Let $\mu: A^{+} \rightarrow\left(S(L), \leq_{L}\right)$ be the syntactic morphism of $L$ Since $\varphi$ recognizes $L$, there exists an onto monotonous morphism $\psi:(S, \leq) \rightarrow\left(S(L), \leq_{L}\right)$ such that $\psi \circ \varphi=\mu$. In particular, it follows that $\left(S(L), \leq_{L}\right) \in \mathbf{V}$. Thus $\mathbf{W} \subseteq \mathbf{V}$.

For the converse, we use the fact that each pseudovariety of finite ordered semigroups is generated by the syntactic ordered semigroups it contains (Lemma 5.1). Let $L \subseteq A^{+}$ be a language whose syntactic ordered semigroup lies in $\mathbf{V}$ and let $\mu: A^{+} \rightarrow\left(S(L), \leq_{L}\right)$ be its syntactic morphism. Then $P=\mu(L)$ is an order ideal of $\left(S(L), \leq_{L}\right)$ and $L=\mu^{-1}(P)$. But $U_{A, \mu} \in \mathcal{U}(\mathbf{V})_{A}$ and $L$ is a union of $U_{A, \mu}$-blocks which forms a $\preceq \mathcal{U}$ order ideal. So $\left(S(L), \leq_{L}\right) \in \mathbf{W}$. It follows that $\mathbf{V} \subseteq \mathbf{W}$, which concludes the proof that $\mathbf{V}(\mathcal{U}(\mathbf{V}))=\mathbf{V}$.

To complete the proof of the corollary, we need to show that $\mathcal{U}(\mathbf{V}(\mathcal{U}))=\mathcal{U}$ for each variety of quasi-uniformities $\mathcal{U}$. What we just showed implies that $\mathbf{V}(\mathcal{U}(\mathbf{V}(\mathcal{U})))=\mathbf{V}(\mathcal{U})$. But the correspondence $\mathcal{U} \mapsto \mathbf{V}(\mathcal{U})$ is one-to-one, so the required equality follows immediately.

We will see examples of this correspondence in Section 5.6.

\subsection{Varieties of quasi-écarts}

We already know (by Corollary 4.8) that each totally bounded and transitive quasiuniformity on a finitely generated semigroup for which multiplication is uniformly continuous, can be described by an ultrametric quasi-écart. Let $\mathbf{V}$ be a pseudovariety of 
ordered semigroups. We now give an explicit description of a quasi-écart defining the quasi-uniformity $\mathcal{U}(\mathbf{V})_{A}$ on some finitely generated free semigroup $A^{+}$.

For all $u, v \in A^{+}$, we say that an ordered semigroup $S$ separates $u$ and $v$ if there exists a morphism $\varphi: A^{+} \rightarrow S$ such that $\varphi(u) \not \leq \varphi(v)$. Now we set

$$
r_{\mathbf{V}}(u, v)=\min \{|S| \mid S \in \mathbf{V}, S \text { separates } u \text { and } v\}
$$

for all $u, v \in A^{+}\left(r_{\mathbf{V}}(u, v)=\infty\right.$ if no semigroup in $\mathbf{V}$ separates $u$ and $\left.v\right)$. Finally, we let

$$
e_{\mathbf{V}}(u, v)=2^{-r_{\mathbf{V}}(u, v)}
$$

with the convention that $2^{-\infty}=0$. Then $e_{\mathbf{V}}$ is an ultrametric quasi-écart.

Observe that there are only finitely many semigroups with a given finite cardinality (up to isomorphism). This implies easily that the quasi-écart $e_{\mathbf{V}}$ is precompact and that the quasi-uniformity defined by $e_{\mathbf{V}}$ on $A^{+}$is $\mathcal{U}(\mathbf{V})_{A}$.

This in turn leads to the following definitions and statements, which the reader may find more palatable than those of the previous sections.

A variety of quasi-écarts is a family $\mathcal{E}=\left(e_{A}\right)_{A}$ of precompact, ultrametric quasiécarts defined on each free semigroup $A^{+}$( $A$ finite), such that the multiplication on $A^{+}$is uniformly continuous, and such that the morphisms between free semigroups are uniformly continuous. We say that two varieties of quasi-écarts $\mathcal{E}=\left(e_{A}\right)_{A}$ and $\mathcal{E}^{\prime}=\left(e_{A}^{\prime}\right)_{A}$ are (uniformly) equivalent if for each alphabet $A$, the quasi-écarts $e_{A}$ and $e_{A}^{\prime}$ are uniformly equivalent.

For instance, if $\mathbf{V}$ is a pseudovariety of ordered monoids, the collection $\mathcal{E}(\mathbf{V})$ of all the quasi-écarts $e_{\mathbf{V}}$ defined on the free semigroups $A^{+}$( $A$ finite), forms a variety of quasiécarts.

Since the quasi-uniformities in a variety can be defined by a precompact ultrametric quasi-écart, there is a natural bijective correspondence between varieties of quasiuniformities and equivalence classes of varieties of quasi-écarts. This correspondence leads to a bijective correspondence between pseudovarieties of ordered semigroups and equivalence classes of varieties of quasi-écarts.

In one direction, the correspondence associates with a pseudovariety of ordered semigroups $\mathbf{V}$, the equivalence class of the variety $\mathcal{E}(\mathbf{V})$. To describe the correspondence in the reverse direction, we observe that if $(S, \mathcal{U})$ is a finitely generated quasi-uniform semigroup such that $\mathcal{U}$ is defined by a precompact ultrametric quasi-écart $e$, then the balls $B_{e}\left(x_{0}, \varepsilon\right)=\left\{x \in X \mid \max \left(e\left(x, x_{0}\right), e\left(x_{0}, x\right)\right)<\varepsilon\right\}(\varepsilon>0)$ are $\mathcal{U}$-blocks, and hence are recognizable subsets of $S$. If $\mathcal{E}=\left(e_{A}\right)_{A}$ is a variety of quasi-écarts, we let $\mathbf{V}(\mathcal{E})$ be the pseudovariety of ordered semigroups generated by the syntactic ordered semigroups of the $B_{e}\left(x_{0}, \varepsilon\right)\left(x_{0} \in X, \varepsilon>0\right)$.

With the above notation, the results of the previous sections can be rewritten as follows.

Theorem 5.7 The correspondences $\mathbf{V} \mapsto \mathcal{E}(\mathbf{V})$ and $\mathcal{E} \mapsto \mathbf{V}(\mathcal{E})$ induce mutually reciprocal bijective correspondences between pseudovarieties of ordered semigroups and equivalence classes of quasi-écarts.

\subsection{Varieties of uniformities}

It is worth stating explicitly the results concerning the special case of uniformities. We already observed that pseudovarieties of semigroups are special cases of pseudovarieties of ordered semigroups. Similarly, we define a variety of uniformities $\mathcal{U}=\left(\mathcal{U}_{A}\right)_{A}$ to be a variety of quasi-uniformities such that each $\mathcal{U}_{A}$ is a uniformity. 
The behavior of the correspondence defined in Section 5.1 when restricted to pseudovarieties of semigroups and varieties of uniformities, is described in the following result.

Theorem 5.8 The correspondences $\mathbf{V} \mapsto \mathcal{U}(\mathbf{V})$ and $\mathcal{U} \mapsto \mathbf{V}(\mathcal{U})$ are mutually reciprocal bijective correspondences between pseudovarieties of semigroups and varieties of uniformities.

Proof. It suffices to show that if $\mathbf{V}$ is a pseudovariety of semigroups, then $\mathcal{U}(\mathbf{V})$ is a variety of uniformities, and that if $\mathcal{U}$ is a variety of uniformities, then $\mathbf{V}(\mathcal{U})$ is a pseudovariety of semigroups.

First let us consider a variety of uniformities $\mathcal{U}$. For each alphabet $A$, the quasiorder $\preceq \mathcal{U}_{A}$ is a congruence on $A^{+}$, and any $\mathcal{U}_{A^{-}}$block is a $\preceq \mathcal{U}_{A}$-order ideal. In particular, the complement of a $\mathcal{U}_{A}$-block also is a $\preceq \mathcal{U}_{A}$-order ideal. Thus, the pseudovariety $\mathbf{V}(\mathcal{U})$ is generated by the ordered syntactic semigroups of the $\mathcal{U}_{A}$-blocks. Let $B$ be such a block. It is easily verified that the ordered syntactic semigroup of the complement of $B$ is $\left(S(B), \geq_{B}\right)$, that is, it is isomorphic to $S(B)$ as a semigroup, and it is equipped with the reverse order of the syntactic order of $B$. So for each generator $(S, \leq)$ of $\mathbf{V}(\mathcal{U})$, we also have $(S, \geq) \in \mathbf{V}(\mathcal{U})$. But the semigroup $(S,=)$ is isomorphic to a subsemigroup of the direct product $(S, \leq) \times(S, \geq)$, so $\mathbf{V}(\mathcal{U})$ is in fact generated by semigroups of the form $(S,=)$, and hence it is a pseudovariety of semigroups.

Now we consider a pseudovariety of semigroups $\mathbf{V}$. For each element $(S, \leq) \in \mathbf{V}$, the ordered semigroup $(S,=)$ also lies in $\mathbf{V}$, so a basis of $\mathcal{U}(\mathbf{V})_{A}$ is given by the $\bigcup_{s \in S} \varphi^{-1}(s) \times$ $\varphi^{-1}(s)$, where $\varphi: A^{+} \rightarrow S$ is a morphism into an element $S$ of $\mathbf{V}$. But each such entourage is symmetrical, so $\mathcal{U}(\mathbf{V})_{A}$ is a uniformity. This concludes the proof.

If $\mathbf{V}$ is a pseudovariety of semigroups, the quasi-écart $e_{\mathbf{V}}$ defined in Section 5.3 is an écart. Naturally, an analogue of Theorem 5.8 can be given, linking pseudovarieties of semigroups and equivalence classes of écarts as in Theorem 5.7.

Finally, let us observe that the écart $e_{\mathbf{V}}$ is a distance function if and only if, for each alphabet $A$, any two distinct words $u, v \in A^{+}$are separated by a semigroup in $\mathbf{V}$, that is, if and only if the free semigroups are residually $\mathbf{V}$.

\subsection{Quasi-uniformities arising from a variety}

Before we give specific examples of the correspondence established in Corollary 5.6 and Theorem 5.7, we will answer the question posed in the introduction of this paper; namely we will characterize those distances on the free semigroup which occur in some variety of écarts, and more generally, those quasi-uniformities on free semigroups which occur in a variety of quasi-uniformities. We prove the following result.

Theorem 5.9 Let $A$ be an alphabet, and let $\mathcal{U}$ be a quasi-uniformity on $A^{+}$. There exists a variety of quasi-uniformities $\mathcal{V}$ such that $\mathcal{U}=\mathcal{V}_{A}$ if and only if $\mathcal{U}$ is totally bounded and transitive, and the multiplication and the endomorphisms of $A^{+}$are $\mathcal{U}$-uniformly continuous.

In terms of quasi-écarts, the corresponding statement is the following.

Corollary 5.10 Let $A$ be an alphabet, and let e be a quasi-écart on $A^{+}$. There exists a variety of quasi-écarts $\mathcal{E}=\left(e_{B}\right)_{B}$ such that $e=e_{A}$ if and only if $e$ is ultrametric and precompact, and the multiplication and the endomorphisms of $A^{+}$are e-uniformly continuous. 
The proof of these results will be achieved by using the language characterization of pseudovarieties of semigroups, that is, by using Eilenberg's original variety theorem (Pin's theorem for pseudovarieties of ordered semigroups).

Let us recall a few definitions and results (see $[8,12,13]$ ). If $K, L \subseteq A^{+}$, the residuals $K^{-1} L$ and $L K^{-1}$ are defined by

$$
\begin{aligned}
& K^{-1} L=\left\{v \in A^{+} \mid \exists u \in K, u v \in L\right\} \\
& L K^{-1}=\left\{v \in A^{+} \mid \exists u \in K, v u \in L\right\} .
\end{aligned}
$$

A class of languages is a correspondence $\mathcal{W}$ which associates with each alphabet $A$ a set $A^{+} \mathcal{W}$ of languages of $A^{+}$. A variety (resp. positive variety) of languages is a class of languages $\mathcal{W}$ such that, for each alphabet $A, A^{+} \mathcal{W}$ is a Boolean algebra of rational languages (resp. a class of rational languages closed under finite union and intersection), closed under taking residuals, and such that, for each morphism $\varphi: A^{+} \rightarrow B^{+}$between free semigroups, $L \in B^{+} \mathcal{W}$ implies $\varphi^{-1}(L) \in A^{+} \mathcal{W}$. The pseudovariety of semigroups (resp. ordered semigroups) associated with the variety (resp. positive variety) of languages $\mathcal{W}$ is the pseudovariety generated by the syntactic (resp. syntactic ordered) semigroups of the elements of the $A^{+} \mathcal{W}$, where $A$ runs over all alphabets. Conversely, one can associate with each such pseudovariety $\mathbf{W}$ the class of languages $\mathcal{W}^{\prime}$ such that, for each alphabet $A, A^{+} \mathcal{W}^{\prime}$ consists of all the languages in $A^{+}$whose syntactic (resp. syntactic ordered) semigroup is in W. Then Eilenberg's variety theorem (Pin's positive variety theorem for ordered semigroups) states that these are one-to-one, onto and mutually reciprocal correspondences, between the class of all pseudovarieties of semigroups (resp. ordered semigroups) and the class of all varieties (resp. positive varieties) of languages.

Because the intersection of a family of (positive) varieties of languages is again a (positive) variety, one can define unequivocally the (positive) variety defined by a given family $\mathcal{C}$ of languages. It is also the (positive) variety of languages associated with the pseudovariety of (ordered) semigroups generated by the syntactic (ordered) semigroups of the languages in $\mathcal{C}$. We will use the following result.

Proposition 5.11 Let $\mathcal{C}$ be a set of rational languages in a given free semigroup $A^{+}$. Let $\mathcal{V}$ be the variety (resp. positive variety) of languages generated by $\mathcal{C}$. Let $B$ be an alphabet. Then $B^{+} \mathcal{V}$ is the closure under the taking of residuals and under Boolean operations (resp. union and intersection) of the set of languages of the form $\varphi^{-1}(L)$, where $L \in \mathcal{C}$ and $\varphi: B^{+} \rightarrow A^{+}$is a morphism.

Proof. We give the proof only in the case of a variety. The proof in the positive variety case is entirely similar.

For each alphabet $B$, let $B^{+} \mathcal{W}$ be the closure under the taking of residuals and under Boolean operations of the set of languages of the form $\varphi^{-1}(L)$, where $L \in \mathcal{C}$ and $\varphi: B^{+} \rightarrow$ $A^{+}$is a morphism. By definition of $\mathcal{W}, \mathcal{C}$ is contained in $A^{+} \mathcal{W}$, and by definition of a variety of languages, $B^{+} \mathcal{W} \subseteq B^{+} \mathcal{V}$. So it suffices to show that $\mathcal{W}$ is a variety of languages. In view of the definition of $\mathcal{W}$, this reduces to verifying that if $\psi: C^{+} \rightarrow B^{+}$is a morphism and if $L \in B^{+} \mathcal{W}$, then $\psi^{-1}(L) \in C^{+} \mathcal{W}$.

But it is easy to verify that the inverse morphism $\psi^{-1}$ commutes with the Boolean operations and the taking of residuals. Thus $\psi^{-1}(L)$ is obtained by means of Boolean operations and by taking residuals, starting from languages of the form $\psi^{-1} \varphi^{-1}\left(L^{\prime}\right)$, where $L^{\prime} \in \mathcal{C}$ and $\varphi: B^{+} \rightarrow A^{+}$is a morphism. To complete the proof, it suffices to observe that $\psi^{-1} \varphi^{-1}=(\varphi \circ \psi)^{-1}$ and that $\varphi \circ \psi$ is a morphism from $C^{+}$into $A^{+}$. 
Let us now prove Theorem 5.9.

Proof of Theorem 5.9. Let $\mathcal{U}$ be a totally bounded, transitive quasi-uniformity on the free semigroup $A^{+}$such that the multiplication and the endomorphisms of $A^{+}$are $\mathcal{U}$ uniformly continuous. Let $\mathcal{C}$ be the set of languages of $A^{+}$which are unions of $\mathcal{U}$-blocks and form $\preceq$-ideals, and let $\mathcal{W}$ be the variety of languages generated by $\mathcal{C}$. Finally, let $\mathbf{V}$ be the pseudovariety of ordered semigroups associated with $\mathcal{W}$ and let $\mathcal{U}(\mathbf{V})$ be the corresponding variety of quasi-uniformities. We want to show that $\mathcal{U}=\mathcal{U}(\mathbf{V})_{A}$.

Let $U$ be an entourage of $\mathcal{U}$, and let $B_{1}, \ldots, B_{n}$ be the $U$-blocks. For each $1 \leq j \leq n$, let $\downarrow B_{j}$ be the $\preceq \mathcal{U}$-order ideal generated by $B_{j}$, and let $\preceq_{j}$ be the syntactic quasi-order of $\downarrow B_{j}$. By definition, each $\downarrow B_{j}$ is in $\mathcal{C}$, and hence the quasi-order $\preceq_{j}$ lies in $\mathcal{U}(\mathbf{V})_{A}$. Now we have

$$
U=\bigcup_{j}\left(\downarrow B_{j}\right) \times B_{j},
$$

so it is easily verified that if $u, v \in A^{+}$and $u \preceq_{j} v$ for each $j$, then $(u, v) \in U$. That is, $U$ contains the intersection of the $\preceq_{j}$, and hence $U \in \mathcal{U}(\mathbf{V})_{A}$. Thus, $\mathcal{U} \subseteq \mathcal{U}(\mathbf{V})_{A}$.

Now let us prove the converse containment. A basis of $\mathcal{U}(\mathbf{V})_{A}$ is given by the finite intersections of entourages of the form $U_{\sigma}$, where $\sigma: A^{+} \rightarrow\left(S(L), \leq_{L}\right)$ is the syntactic morphism of a language $L$ of $A^{+} \mathcal{V}$. By Proposition 5.11, $L$ is obtained from languages of the form $\varphi^{-1}\left(L^{\prime}\right)\left(L^{\prime} \in \mathcal{C}, \varphi\right.$ an endomorphism of $\left.A^{+}\right)$by means of unions, intersections and residuals. Now we observe that if $L=\bigcup_{i=1}^{n} L_{i}$ or $L=\bigcap_{i=1}^{n} L_{i}$, and if $\tau_{i}$ is the syntactic morphism of $L_{i}(1 \leq i \leq n)$, then $U_{\sigma}$ contains $\bigcap_{i=1}^{n} U_{\tau_{i}}$; similarly, if $L=K^{-1} L^{\prime}$ and if $\tau^{\prime}$ is the syntactic morphism of $L^{\prime}$, then $U_{\sigma}$ contains $U_{\tau^{\prime}}$; finally, if $\varphi$ is an endomorphism of $A^{+}$, if $L=\varphi^{-1}\left(L^{\prime}\right)$, and if $\tau^{\prime}$ is the syntactic morphism of $L^{\prime}$, then $U_{\sigma}$ contains $\varphi^{-1}\left(U_{\tau^{\prime}}\right)$. Using the fact that each endomorphism of $A^{+}$is $\mathcal{U}$-uniformly continuous, it follows that $U_{\sigma} \in \mathcal{U}$, that is, $\mathcal{U}(\mathbf{V})_{A} \subseteq \mathcal{U}$.

Example 5.12 Let $A=\{a, b\}$. The syntactic congruence $\sim$ of the language $\{a b a b\}$ has 8 classes: $\{a\},\{b\},\{a b\},\{b a\},\{a b a\},\{b a b\},\{a b a b\}$, and the complement in $A^{+}$of the 7 previous classes. Let $e$ be the corresponding écart on $A^{+}$, that is,

$$
e(u, v)= \begin{cases}0 & \text { if } u \sim v \\ 1 & \text { otherwise }\end{cases}
$$

for all $u, v \in A^{+}$.

Then Theorem 5.9 shows that $e$ cannot occur in a variety of écarts. Indeed, $e$ is ultrametric and precompact, as can be easily verified; the multiplication of $A^{+}$is uniformly continuous for $e$; but the endomorphism $\varphi$ of $A^{+}$given by $\varphi(a)=a b$ and $\varphi(b)=b b$, is not continuous: $\{a b a b\}$ is an $e$-ball of radius $1 / 2$, and $\varphi^{-1}(a b a b)=\left\{a^{2}\right\}$ does not contain any $e$-ball.

\subsection{Examples}

First let us note that everything we did for quasi-uniform structures on free semigroups $A^{+}$ and for pseudovarieties of ordered semigroups, can be done in the same fashion for quasiuniform structures on free monoids $A^{*}$ and for pseudovarieties of ordered monoids. Some of the examples below will be of varieties of quasi-uniform structures on free monoids.

To compute examples, we will use some information on the languages recognized by ordered semigroups in given pseudovarieties, and the result of Lemma 5.3: the finite intersections of syntactic quasi-orders of languages recognized by elements of $\mathbf{V}$ form a basis of $\mathcal{U}(\mathbf{V})_{A}$. 


\section{A distance based on prefixes}

If $u, v$ are words in $A^{+}$, we let $p(u, v)$ be the longest common prefix of $u$ and $v$, and we let

$$
d(u, v)= \begin{cases}0 & \text { if } u=v, \\ 2^{-|p(u, v)|} & \text { if } u \neq v .\end{cases}
$$

Then $d$ defines an ultrametric, precompact distance on $A^{+}$, which defines a uniformity $\mathcal{U}$ on $A^{+}$. The collection of these uniformities on the free semigroups forms a variety of uniformities. Note that for each $n \geq 1$, we have

$$
U_{2^{-n}}=\left\{(u, v) \mid d(u, v)<2^{-n}\right\}=\bigcup_{w \in A^{n}} w A^{*} \times w A^{*} .
$$

Thus the $U_{2^{-n}}$-blocks are the languages of the form $w A^{*}$ for some word $w$ of length $n$. It is well known [12] that the syntactic semigroups of these languages generate the pseudovariety of semigroups $\mathbf{K}$, which consists of those semigroups $S$ in which $e S=e$ for each idempotent $e$. Thus we have $\mathbf{V}(\mathcal{U})=\mathbf{K}$.

\section{Another distance based on prefixes}

Another distance based on prefixes has been commonly used in automata theory (see for instance Choffrut [5]). It is given by

$$
d(u, v)=|u|+|v|-2|p(u, v)|
$$

for all $u, v \in A^{+}$. This distance does not fit within our framework, that is, it is not uniformly equivalent to an écart occurring in a variety of écarts. Indeed, $d$ is not ultrametric: if $a, b \in A$, then we have $d(a, b)=2, d\left(b, b^{2}\right)=1$ and $d\left(a, b^{2}\right)=3$, which is greater than $\max (1,2)$. However, the multiplication in $A^{+}$and the morphisms between free semigroups are uniformly continuous. This is due to the fact that every $d$-ball of small enough radius (say, of radius less than $1 / 2$ ) is a singleton. The same property would hold for any distance $d^{\prime}$ in which 0 is not a limit point of the set of values of $d^{\prime}$, such as $d^{\prime}=d /(1+d)$.

\section{A quasi-écart based on subwords}

In Example 2.2 and in subsequent examples, we have studied the subword quasi-order $\leq_{\mathrm{sw}}$ and the subword quasi-uniformity. If $u, v \in A^{*}$ and $v$ is not a subword of $u$, we let $r(u, v)$ be the minimal length of a word $w$ which is a subword of $v$ and not a subword of $u$. We let

$$
e(u, v)= \begin{cases}0 & \text { if } v \text { is a subword of } u, \\ 2^{-r(u, v)} & \text { otherwise. }\end{cases}
$$

One verifies that $e$ is an ultrametric, precompact quasi-écart on $A^{*}$. Let $\mathcal{U}$ be the quasiuniformity on $A^{*}$ defined by $e$. The collection of these quasi-uniformities forms a variety. By definition, for each $n>0, U_{2^{-n}}$ is set of all pairs of words $(u, v)$ such that every subword of $v$ of length at most $n$ is also a subword of $u$ : it is the intersection of the $U_{x}$ where $x$ runs over all words of length at most $n$, that is, the intersection of the syntactic quasi-orders of the $L_{x}$ for these words. In particular, the quasi-uniformity defined by $e$ is exactly the subword quasi-uniformity.

Let $\mathbf{J}^{+}$be the pseudovariety of ordered monoids which consists of all ordered monoids in which 1 is the maximal element. It is known [13] that a language is recognized by an ordered monoid in $\mathbf{J}^{+}$if and only if it is a finite union of languages of the form $L_{x}$. In view of Lemma 5.3, it follows that the quasi-uniformity $\mathcal{U}$ defined by the quasi-écart $e$ is 
contained in $\mathcal{U}\left(\mathbf{J}^{+}\right)$. To prove the converse inclusion, let us consider the basis of $\mathcal{U}\left(\mathbf{J}^{+}\right)$ formed by the syntactic quasi-orders $\preceq_{L_{x}}$ of the languages of the form $L_{x}, x \in A^{+}$. If $n=|x|$, then $U_{2^{-n}}$ is contained in ${ }_{L_{x}}$, so $\preceq_{L_{x}}$ is an entourage of $\mathcal{U}$. Thus $\mathcal{U}=\mathcal{U}\left(\mathbf{J}^{+}\right)$, or equivalently, $\mathbf{V}(\mathcal{U})=\mathbf{J}^{+}$. In particular, the quasi-order $\preceq \mathbf{J}^{+}$coincides with the subword order $\leq_{\text {sw }}$ on $A^{*}$.

Let us now consider the symmetrized version of the above quasi-écart. It is given by $d(u, v)=\max (e(u, v), e(v, u))$. Observe that $d$ is a distance, and $d(u, v)<2^{-n}$ if $u$ and $v$ have the same subwords of length at most $n$. The corresponding pseudovariety of monoids, $\mathbf{V}\left(\mathcal{U}^{*}\right)$, is generated by the monoids of the form $(S,=)$ such that $(S, \leq) \in \mathbf{J}^{+}$for some order $\leq$. By a result of Straubing and Thérien [18] (or equivalently, by Simon's theorem on piecewise testable languages, see [12]), $\mathbf{V}\left(\mathcal{U}^{*}\right)$ is the pseudovariety $\mathbf{J}$ of $\mathcal{J}$-trivial monoids, that is, of monoids in which distinct elements generate distinct two-sided ideals.

\section{A distance based on factors}

Let $u, v$ be words. We say that $v$ is a factor of $u$ if there exist words $x, y \in A^{*}$ such that $u=x v y$. The set of factors of length at most $k$ of $u$ is denoted by $F_{k}(u)$. We also let $p_{k}(u)$ and $s_{k}(u)$ be the words defined as follows.

$$
\begin{aligned}
& p_{k}(u)= \begin{cases}u & \text { if }|u|<k, \\
\text { the prefix of } u \text { of length } k & \text { otherwise; }\end{cases} \\
& s_{k}(u)= \begin{cases}u & \text { if }|u|<k, \\
\text { the suffix of } u \text { of length } k & \text { otherwise }\end{cases}
\end{aligned}
$$

If $u$ and $v$ are words in $A^{+}$, we let $r(u, v)$ be the least integer $k$ such that, either $p_{k-1}(u) \neq$ $p_{k-1}(v)$, or $s_{k-1}(u) \neq s_{k-1}(v)$, or $F_{k}(v) \nsubseteq F_{k}(u)$. Finally, we let $e(u, v)=2^{-r(u, v)}$.

Again, one verifies that $e$ is a precompact, ultrametric quasi-écart on $A^{+}$, and that the collection of these quasi-écarts forms a variety. Let $\mathcal{U}$ be the quasi-uniformity defined by $e$ on $A^{+}$. For each $n>0$, the entourage $U_{2^{-n}}$ of $\mathcal{U}$ is the intersection of the syntactic quasi-orders of the languages of the form $\{x\}, x A^{*}$ and $A^{*} x\left(x \in A^{+},|x|<n\right)$ and of the languages of the form $A^{*} x A^{*}\left(x \in A^{+},|x| \leq n\right)$. Observe in particular that the quasi-order $\preceq \mathcal{U}$ on $A^{+}$is trivial.

Let $\mathbf{L J}_{1}^{+}$be the pseudovariety of ordered semigroups $(S, \leq)$ in which $e S e$ is an idempotent and commutative semigroup, and ese $\leq e$ for each idempotent $e$ and for each element $s$. It is known that a language is recognized by an element of $\mathbf{L} \mathbf{J}_{1}^{+}$if and only if it is a positive Boolean combination of languages of the form $\{x\}, x A^{*}, A^{*} x$ and $A^{*} x A^{*}$ $\left(x \in A^{+}\right)$[14]. By the same reasoning as in the previous example, one can show that the pseudovariety of finite ordered semigroups associated with the variety of quasi-écarts defined above is $\mathbf{L} \mathbf{J}_{1}^{+}$. In particular, the quasi-order $\preceq_{\mathbf{L} \mathbf{J}_{1}^{+}}$is trivial.

\section{References}

[1] J. Almeida. Finite Semigroups and Universal Algebra, World Scientific, Singapore, 1994.

[2] J. Almeida and P. Weil. Relatively free profinite monoids: an introduction and examples, in J. Fountain ed. Semigroups, Formal Languages and Groups, NATO ASI Series C-466, Kluwer Academic, 1995, 73-117.

[3] M.M. Bonsangue, F. van Breugel, J.J.M.M. Rutten. Generalized ultrametric spaces: completion, topology and powerdomains via the Yoneda embedding, Theoretical Computer Science, to appear. 
[4] Bourbaki. Élements de mathématiques, topologie générale, Hermann, Paris, 1971.

[5] Ch. Choffrut. Fonctions rationnelles et distances discrètes in Mots - mélanges offerts à M.P. Schützenberger, M. Lothaire ed., Hermes, Paris, 1990, 314-327.

[6] D. Doitchinov. Another class of completable quasi-uniform spaces, Comp. Rend. Acad. Bulgare Sci. 44, (1990), no. 3, 5-6.

[7] D. Doitchinov. A concept of completeness of quasi-uniform spaces. Topology Appl., 38, (1991), 205-217.

[8] S. Eilenberg. Automata, languages and machines, vol. B, Academic Press, New York, 1976.

[9] P. Fletcher and W. Lindgren. Quasi-uniform spaces, Lect. Notes in Pure and Applied Mathematics 77, Marcel Dekker, 1982.

[10] R. Hunter. Certain finitely generated compact zero-dimensional semigroups, J. Austral. Math. Soc. (Series A) 44 (1988) 265-270.

[11] H. Künzi and G. Brümmer. Sobrification and bicompletion of totally bounded quasiuniform spaces, Math. Proc. Camb. Phil. Soc. 101 (1987) 237-247.

[12] J.-E. Pin. Variétés de langages formels, Masson, Paris, 1984. English translation: Varieties of formal languages, North Oxford, London, and Plenum, New York, 1986.

[13] J.-E. Pin. A variety theorem without complementation, Russian Mathematics (Izvestija VUZ Matematika) 39 (1995), 80-90.

[14] J.-E. Pin. Syntactic semigroups, in Handbook of formal languages, G. Rozenberg and A. Salomaa eds., Springer (1997).

[15] J. Reiterman. The Birkhoff theorem for finite algebras, Algebra Universalis 14, (1982), 1-10.

[16] J.J.M.M. Rutten. Elements of generalized ultrametric domain theory, Theoretical Computer Science 170 (1996) 349-381.

[17] A. K. Seda. Quasi-metrics and fixed points in computing, Bulletin of the EATCS 60 (1996) 154-163.

[18] H. Straubing and D. Thérien. Partially ordered finite monoids and a theorem of I. Simon, J. Algebra 119 (1985) 393-399.

[19] P. Sünderhauf. The Smyth-completion of a quasi-uniform space. In M. Droste and Y. Gurevich, eds, Semantics of Programming Languages and Model Theory, volume 5 of Algebra, Logic and Applications, Gordon and Breach Science Publ., Montreux, 1993, 189-212.

[20] P. Sünderhauf. Quasi-uniform completeness in terms of Cauchy nets, Acta Mathematica Hungarica 69 (1995) 47-54.

[21] P. Sünderhauf. A faithful computational model of the real numbers, Theoretical computer Science 151 (1995) 277-294. 